\title{
On the spectrum of mid-latitude sporadic- $E$ irregularities
}

\author{
Yu. V. Kyzyurov \\ Main Astronomical Observatory NASU, Kiev-127, 03680, Ukraine \\ Received: 23 August 1999 / Revised: 7 February 2000 / Accepted: 23 May 2000
}

\begin{abstract}
We discuss the creation of mid-latitude sporadic- $E$ plasma irregularities (with length-scales smaller than sporadic layer thickness) by the neutral atmosphere turbulence. Using fluid equations, the relation between plasma density fluctuations and the velocity field of neutrals is derived. After a brief discussion of the relevant neutral turbulence, the analytical expression for the power spectrum of plasma irregularities is obtained. This expression allows a power-law type of experimental irregularity spectra (the spectral index depends on sporadic- $E$ characteristics) and possible departures in detail of the irregularity spectra from the power-law form to be explained. In addition, it allows us to make estimates of length-scales at which such departures must occur.
\end{abstract}

Key words: Ionosphere (ionospheric irregularities; midlatitude ionosphere) - Space plasma physics (turbulence)

\section{Introduction}

The most widely accepted explanation for mid-latitude sporadic- $E\left(E_{s}\right)$ is the windshear theory. According to this theory, the sporadic- $E$ layer is caused by a redistribution of ionization through the interaction of the plasma imbedded in the neutral winds with the geomagnetic field under the appropriate vertical profile of the horizontal wind velocity. The first detailed discussions of such a formation mechanism for $E_{s}$-layers at middle latitudes were presented by Whitehead (1961) and Axford (1963). At the present time, there are a large number of papers on the theory and observation of midlatitude sporadic- $E$ (e.g., see reviews by Whitehead, 1970, 1989, and a monograph by Gershman et al.,

Correspondence to: $\mathrm{Yu}$. V. Kyzyurov

e-mail:kyzyurov@mao.kiev.ua
1976). Direct measurements of winds and electron density profiles support the windshear theory. Thus, shear flows in the upper atmosphere ( $E$-region altitudes) can produce $E_{s}$. On the other hand, under certain conditions the shear flow in fluids (or gas) can be turbulent (see e.g., Townsend, 1976). Therefore, the neutral air turbulence must play an important part in the dynamics of sporadic- $E$ layers, if a sporadic- $E$ height is not above the turbopause. Chimonas (1974) investigated the effect of turbulence on a sporadic- $E$ layer and showed, in particular, that development of turbulence causes a decrease in electron density and an increase in thickness of the layer. The role of the neutral atmosphere turbulence in the creation of sporadic- $E$ irregularities is discussed in a monograph by Gershman et al. (1976); however, the spectrum of such irregularities is not treated. In some papers concerning irregularities in the lower ionosphere, it is asserted (often without sufficient physical arguments) that the spectrum of these irregularities simply coincides with that of the neutral atmosphere turbulence (e.g., Teptin and Stenin, 1977). Data of observations show that the spatial (or wave number) spectrum of ionospheric irregularities can be described by a power-law form $P(k) \propto k^{-d}(d>0)$ and in some cases $d \approx 1.67$ indeed, however, on the other hand, the spectral shape can differ from a power-law shape with a constant index, e.g., $d$ ranged from 0.8 to 3 for $E_{s}$ irregularities with length-scales about $100-400 \mathrm{~m}$ (Ovezgeldyev et al., 1988).

Our purpose is to consider the possibility of plasma irregularity formation by the neutral atmosphere turbulence in the mid-latitude sporadic- $E$ and to obtain the spectrum of such irregularities.

Recently, an important step towards better understanding of the nature of plasma irregularities caused by neutral turbulence in the lower $E$-region was made by Gurevich et al. (1997) and Schlegel and Gurevich (1997). Using a fluid approximation, the spectrum of induced fluctuations of electron and ion densities and electric field was derived in the first paper. The cross section for scattering of radio waves from the lower ionosphere was also determined there. This cross section 
was quantitatively explored and the implications for backscatter experiments in the MF, HF and VHF ranges were discussed by Schlegel and Gurevich (1997). Unlike the work by Gurevich et al. (1997) in which the ionospheric turbulence induced by the turbulent motion of neutral gas is presented as the superposition of random plasma waves and investigated in application to the lower part of the $E$-region, a situation with conventional fluid turbulence creating irregularities through mixing of plasma in the mid-latitude sporadic- $E$ will be considered here.

It should be noted that sporadic- $E$ irregularities are produced by plasma instabilities when the altitude of the layer is above the turbopause (Whitehead, 1993). There are a number of papers about plasma instabilities in the mid-latitude sporadic- $E$ (Ecklund et al., 1981; Riggin et al., 1986; Woodman et al., 1991; Whitehead, 1993). A detailed discussion of recent studies of these instabilities was presented by Haldoupis and Schlegel (1993). It is interesting that both the gradient drift instability and the two-stream (FarleyBuneman) instability can operate in the mid-latitude ionospheric E-region (Haldoupis and Schlegel, 1993; Schlegel and Haldoupis, 1994; Haldoupis et al., 1997). Both these instabilities are basic mechanisms which generate plasma irregularities in the equatorial and auroral electrojets at ionospheric $E$-region altitudes (for details, see Fejer and Kelley, 1980; Farley, 1985; Haldoupis, 1989; Whitehead, 1989, and references therein). The analytical and numerical studies based on the plasma turbulence theory explain the main features of the electrojet irregularities (Sudan, 1983; Nielsen and Schlegel, 1985; Robinson, 1986; Ronchi et al., 1991; Hamza and St-Maurice, 1993a, b; Schlegel and Thiemann, 1994; Hamza and Sudan, 1995). Ponyatov (1988) has applied the approach, developed by Sudan (1983) and Sudan and Pfirsch (1985), to the mid-latitude $E$-region and obtained the spectrum of the gradient drift instability.

It is known that compared to the auroral and equatorial zones the acting dc electric fields in the midlatitude $E$-region are small, hence, the threshold conditions for the two-stream instability are seldom met at moderate latitudes and the electron density gradient role becomes very important for the excitation of the gradient drift instability. On the other hand, when the neutral atmosphere turbulence destroys the sharp density gradient it must eliminate the favorable conditions for the gradient drift plasma instability as a rule. Thus, the plasma instabilities are beyond our scope.

It needs to be pointed out that the irregularities created by neutral turbulence are quasi-isotropic (Fooks, 1961; Røyrvic and Smith, 1984; Ovezgeldyev et al., 1988), and the gradient drift irregularities are strongly anisotropic, i.e., aligned along the Earth's magnetic field (e.g., see Ecklund et al., 1981; Whitehead, 1989; Schlegel and Haldoupis, 1994). As is known the isotropy of ionospheric irregularities results in the weak aspect sensitivity of radar returns while the field-aligned irregularities provide the strong aspect sensitivity
(Røyrvic and Smith, 1984; Cho et al., 1993; Huang et al., 1995). Moreover, the non-field-aligned irregularities tend to move with the neutral air, while the fieldaligned ones tend to stay fixed to the magnetic field lines (Namboothiri et al., 1994).

\section{Basic equations and assumptions}

The ionospheric plasma can be regarded as consisting of three-components, i.e., it consists of neutrals and two types of charged particles. When the motion of neutrals is given (the neutral gas is assumed to be unaffected by collisions with the ionized component), many processes in the $E$-region ionospheric plasma can be described by fluid equations in the next form (Gershman et al., 1976)

$$
\begin{aligned}
& \partial \mathbf{v}_{\alpha} / \partial t+\left(\mathbf{v}_{\alpha} \nabla\right) \mathbf{v}_{\alpha}+v_{\alpha}\left(\mathbf{v}_{\alpha}-\mathbf{u}\right) \\
& \quad=q_{\alpha} \mathbf{E} / m_{\alpha}+\Omega_{\alpha} \mathbf{v}_{\alpha} \times \mathbf{b}-v_{T \alpha}^{2} \nabla n_{\alpha} / n_{\alpha}, \\
& \partial n_{\alpha} / \partial t+\nabla\left(n_{\alpha} \mathbf{v}_{\alpha}\right)=J-\alpha_{\mathrm{r}} n_{\alpha}^{2},
\end{aligned}
$$

where subscript $\alpha$ denotes the species (electron, $\alpha=\mathrm{e}$, or ion, $\alpha=\mathrm{i}), \mathbf{v}_{\alpha}(\mathbf{x}, t)$ is the charged particle velocity, $v_{\alpha}$ is the charged particle neutral collision frequency, $\mathbf{u}(\mathbf{x}, t)$ is the neutral gas velocity, $q_{\alpha}$ is the particle charge $\left(q_{\mathrm{i}}=-q_{\mathrm{e}}=\mathrm{e}\right), m_{\alpha}$ is the particle mass, $\mathbf{E}$ is the electric field, $\mathbf{b}=\mathbf{B} /|\mathbf{B}|$ is the unit vector along the geomagnetic field $\mathbf{B}$, and $\Omega_{\alpha}=q_{\alpha}|\mathbf{B}| / m_{\alpha} c$ is the charged particle gyrofrequency, $\mathrm{v}_{T \alpha}$ is the thermal velocity of the particle, $n_{\alpha}(\mathbf{x}, t)$ is the charged particle number density, $J$ is the rate of production of particles per unit volume and $\alpha_{\mathrm{r}}$ is the recombination coefficient. The effects of collisions between charged particles are neglected. Values of $v_{\alpha}$, $\Omega_{\alpha}, J$, and $\alpha_{\mathrm{r}}$ are presumed to be constant throughout the work.

The processes of interest are rather slow, i.e., the length-scale of the processes is larger than the ion mean free path, $l \gg \Lambda_{\mathrm{i}}$, and the time scale is larger than the inverse value of ion neutral collision frequency, $t \gg v_{i}^{-1}$ (the charged particles undergo many collisions with neutrals during the time of interest). Hence it is possible to neglect the so called "inertia" terms on the left of Eq. (1)

$q_{\alpha} \mathbf{E} / m_{\alpha}+\Omega_{\alpha} \mathbf{v}_{\alpha} \times \mathbf{b}-v_{\alpha}\left(\mathbf{v}_{\alpha}-\mathbf{u}\right)-\mathrm{v}_{T \alpha}{ }^{2} \nabla n_{\alpha} / n_{\alpha}=0$.

It is seen that Eq. (3) represents a balance between the terms from the density gradients and the drag of the background wind against the Lorentz forces. Taking into account that in the $E$-region $v_{\mathrm{i}}>\Omega_{\mathrm{i}}$ and $v_{\mathrm{e}}<\Omega_{\mathrm{e}}$, for slow processes in space and time we may regard $n_{\mathrm{e}} \approx n_{\mathrm{i}}=n$, the only electric field considered is that required to prevent charge separation (due to the field $\mathbf{E}$, electrons tend to follow ions, other background electric fields are ignored), and eliminating this electric field from Eq. (3) we obtain the local drift velocity of ions (or plasma in general)

$\mathbf{v}_{\mathrm{i}}(\mathbf{x}, t) \approx \mathbf{u}(\mathbf{x}, t)+\beta_{\mathrm{i}} \mathbf{u} \times \mathbf{b}-D_{\mathrm{a}} \nabla n / n$, 
where $\beta_{\mathrm{i}}=\Omega_{\mathrm{i}} / v_{\mathrm{i}}, D_{\mathrm{a}} \approx v_{s}^{2} / v_{\mathrm{i}}$ is the ambipolar diffusion coefficient $\left(v_{\mathrm{s}}^{2}=\mathrm{v}_{T \mathrm{i}}^{2}+m_{\mathrm{e}} \mathrm{v}_{T \mathrm{e}}^{2} / m_{\mathrm{i}}\right)$, its dependence on the magnetic field can be ignored in the case of the lower ionosphere (see, e.g., Axford, 1963).

Using Eq. (4), the equation of continuity Eq. (2) then becomes

$\partial n / \partial t+\nabla\left(n\left(\mathbf{u}+\beta_{\mathrm{i}} \mathbf{u} \times \mathbf{b}\right)\right)-D_{\mathrm{a}} \nabla^{2} n=J-\alpha_{\mathrm{r}} n^{2}$,

Such an equation is usually discussed in the windshear theory of $E_{s}$ and allows us to obtain the profile of electron number density under the given profile of neutral gas velocity. In the presence of neutral atmosphere turbulence, the neutral velocity and plasma density fields may be separated into regular (mean) and fluctuating parts:

$\mathbf{u}(\mathbf{x}, t)=\mathbf{u}_{0}(\mathbf{x})+\mathbf{u}_{1}(\mathbf{x}, t), \quad \mathbf{u}_{0}(\mathbf{x})>\mathbf{u}_{1}(\mathbf{x}, t)$,

$n(\mathbf{x}, t)=n_{0}(\mathbf{x})+n_{1}(\mathbf{x}, t), \quad n_{0}(\mathbf{x})>n_{1}(\mathbf{x}, t)$

(we shall regard that $\left\langle\mathbf{u}_{1}\right\rangle=0,\left\langle n_{1}\right\rangle=0$, i.e., $\mathbf{u}_{0}=\langle\mathbf{u}\rangle$ and $n_{0}=\langle n\rangle$, the angle brackets indicate an ensemble average; the existence times of the wind shear and the corresponding $E_{s}$ are large compared with time scales of the fluctuating parts of the u-field and the $n$-field, and may therefore be regarded as infinite). Likewise Eq. (5) may be separated into its mean and fluctuating parts:

$$
\begin{aligned}
& \boldsymbol{\nabla}\left(n_{0}\left(\mathbf{u}_{0}+\beta_{\mathrm{i}} \mathbf{u}_{0} \times \mathbf{b}\right)\right)-D_{\mathrm{a}} \nabla^{2} n_{0} \\
&+\left\langle\boldsymbol{\nabla}\left(n_{1}\left(\mathbf{u}_{1}+\beta_{\mathrm{i}} \mathbf{u}_{1} \times \mathbf{b}\right)\right)\right\rangle=J-\alpha_{\mathrm{r}} n_{0}^{2}-\alpha_{\mathrm{r}}\left\langle n_{1}^{2}\right\rangle, \\
&\{\partial / \partial t-D_{\mathrm{a}} \nabla^{2}+2 \alpha_{\mathrm{r}} n_{0}+\nabla\left(\mathbf{u}_{0}+\beta_{\mathrm{i}} \mathbf{u}_{0} \times \mathbf{b}\right) \\
&\left.+\left(\mathbf{u}_{0}+\beta_{\mathrm{i}} \mathbf{u}_{0} \times \mathbf{b}\right) \nabla\right\} n_{1}+\alpha_{\mathrm{r}}\left(n_{1}^{2}-\left\langle n_{1}^{2}\right\rangle\right) \\
&+\nabla\left(n_{1} \cdot \mathbf{u}_{1}-\left\langle n_{1} \cdot \mathbf{u}_{1}\right\rangle\right) \\
&+\beta_{\mathrm{i}} \nabla\left(n_{1} \mathbf{u}_{1} \times \mathbf{b}-\left\langle n_{1} \mathbf{u}_{1} \times \mathbf{b}\right\rangle\right) \\
&=-\nabla\left(n_{0}\left(\mathbf{u}_{1}+\beta_{\mathrm{i}} \mathbf{u}_{1} \times \mathbf{b}\right)\right) .
\end{aligned}
$$

Equation (9) describes the dynamics of plasma density fluctuations excited in sporadic- $E$ by turbulent motions of neutrals. The term $-\nabla\left(n_{0}\left(\mathbf{u}_{1}+\beta_{\mathrm{i}} \mathbf{u}_{1} \times \mathbf{b}\right)\right)$ in Eq. (9) acts as a source term generating the fluctuating field $n_{1}(\mathbf{x}, t)$. Using the assumption of linear relation between the density fluctuations and the random velocity field we can neglect the term $\alpha_{\mathrm{r}}\left(n_{1}^{2}-\left\langle n_{1}^{2}\right\rangle\right)$. Data of observations show that the characteristic vertical scale $L_{0}$ of neutral wind shear leading to the formation of sporadic- $E$ and the thickness $L_{\mathrm{s}}$ of corresponding layer are much smaller than their horizontal scales (Rosenberg and Justus, 1966; Smith, 1966). The layer considered is plane and infinite in horizontal extent. We will consider plasma density fluctuations with length-scales $l$ smaller than $L_{\mathrm{s}}$, hence, restricting attention to incompressible motions of neutrals for which $\nabla \mathbf{u}=0$ and since in order of magnitude $\quad n_{1} \nabla\left(\mathbf{u}_{0}+\beta_{\mathrm{i}} \mathbf{u}_{0} \times \mathbf{b}=O\left(\beta_{\mathrm{i}} u_{0} n_{1} / L_{0}\right),\left(\mathbf{u}_{0}+\beta_{\mathrm{i}}\right.\right.$ $\left.\mathbf{u}_{0} \times \mathbf{b}\right) \nabla n_{1}=O\left(u_{0} n_{1} / l\right), \beta_{i} \nabla\left(n_{1} \mathbf{u}_{1} \times \mathbf{b}-\left\langle n_{1}\left(\mathbf{u}_{1} \times \mathbf{b}\right)\right\rangle\right)=$ $O\left(\beta_{\mathrm{i}} u_{1} n_{1} / l\right)$, and $\beta_{\mathrm{i}}<1, \quad L_{0}>L_{\mathrm{s}}>l, \quad u_{0}>u_{1}$, then $\beta_{i} \boldsymbol{\nabla}\left(n_{1} \mathbf{u}_{1} \times \mathbf{b}-\left\langle n_{1}\left(\mathbf{u}_{1} \times \mathbf{b}\right\rangle\right) \sim n_{1} \boldsymbol{\nabla}\left(\mathbf{u}_{0}+\beta_{\mathrm{i}} \mathbf{u}_{0} \times \mathbf{b}\right) \ll\right.$ $\left(\mathbf{u}_{0}+\beta_{\mathrm{i}} \mathbf{u}_{0} \times \mathbf{b}\right) \nabla n_{1}$. The term $\left(\mathbf{u}_{0}+\beta_{\mathrm{i}} \mathbf{u}_{0} \times \mathbf{b}\right) \nabla n_{1}$ describes the motion of the whole fluctuation pattern with the drift velocity $\mathbf{v}_{\mathrm{d}}=\mathbf{u}_{0}+\beta_{\mathrm{i}} \mathbf{u}_{0} \times \mathbf{b}$ and can be elimi- nated by choosing a moving frame of reference (of course, this convention is valid if $\mathbf{v}_{\mathrm{d}}$ is uniform, in the present situation a length-scale $l$ of irregularities is small enough compared with the scale $L_{0}$ of variation of mean velocity $\mathbf{u}_{0}$ so that $\mathbf{u}_{0}$ and $\mathbf{v}_{\mathrm{d}}$ may be regarded as nearly uniform). Supposing that the gradient length-scale of mean plasma density $L_{\mathrm{g}}=n_{0} /\left|\nabla n_{0}\right|$ is about $L_{\mathrm{s}}$, and since $\nabla \mathbf{u}_{1}=0$, Eq. (9) becomes

$$
\begin{aligned}
& \left\{\partial / \partial t-D_{\mathrm{a}} \nabla^{2}+\tau_{\mathrm{r}}^{-1}\right\} \delta n+\nabla\left(\delta n \cdot \mathbf{u}_{1}-\left\langle\delta n \cdot \mathbf{u}_{1}\right\rangle\right) \\
& \quad=-L_{\mathrm{s}}^{-1}\left(\mathbf{u}_{1} \cdot \mathbf{h}\right)-\beta_{\mathrm{i}} \boldsymbol{\nabla}\left(\mathbf{u}_{1} \times \mathbf{b}\right),
\end{aligned}
$$

where $\delta n(\mathbf{x}, t)=n_{0}^{-1} n_{1}(\mathbf{x}, t), \tau_{\mathrm{r}}=\left(2 \alpha_{\mathrm{r}} n_{0}\right)^{-1}$ is the characteristic time of recombination, $\mathbf{h}=\mathbf{g}+\beta_{\mathrm{i}} \mathbf{b} \times \mathbf{g}, \mathbf{g}=$ $L_{\mathrm{g}} n_{0}^{-1} \nabla n_{0}$ is the unit vector along the gradient of $n_{0}$, in the present case (to the approximation accepted in this consideration) we may regard $\mathbf{h} \approx \mathbf{g}$. The problem of plasma fluctuation formation by the atmosphere turbulence in the lower ionosphere is a problem of passive scalar convection (plasma density is a passive scalar). Since the neutral atmosphere turbulence in the altitude range of interest is approximately isotropic with a large scale of about $5 \mathrm{~km}$ (Justus, 1967) and the sporadic- $E$ thickness is usually not larger than this scale, then crosscorrelations of the velocity and scalar fields, scalar $\rightarrow$ velocity and velocity $\rightarrow$ scalar, are ruled out by the restriction to passive convection and by the symmetry requirements of isotropic turbulence (e.g., see McComb et al., 1992). Hence, $\left\langle\delta n \cdot \mathbf{u}_{1}\right\rangle=0$, and Eq. (10) may be written in the form

$$
\begin{gathered}
\left\{\partial / \partial t-D_{\mathrm{a}} \nabla^{2}+\tau_{\mathrm{r}}^{-1}\right\} \delta n+\nabla\left(\delta n \cdot \mathbf{u}_{1}\right) \\
=-L_{\mathrm{s}}^{-1}\left(\mathbf{u}_{1} \cdot \mathbf{g}\right)-\beta_{\mathrm{i}} \nabla\left(\mathbf{u}_{1} \times \mathbf{b}\right) .
\end{gathered}
$$

This equation is the linearized form of Eq. (9) and describes the process of formation of plasma density fluctuations with scales smaller than the sporadic- $E$ thickness. Here one must distinguish between two situations: random waves when $\boldsymbol{\nabla}\left(\delta n \cdot \mathbf{u}_{1}\right) \ll \partial \delta n / \partial t$, and conventional turbulence when $|\partial \delta n / \partial t|$ and $\left|\nabla\left(\delta n \cdot \mathbf{u}_{1}\right)\right|$ are of the same order of magnitude (the case of random waves in application to plasma density fluctuations caused by neutral turbulence in the lower part of $E$-region was considered by Gurevich et al. 1997, the present work deals with the case of conventional turbulence). It may be seen that the first term on the right-hand side of Eq. (11) is more important at larger length-scales $l>\beta_{\mathrm{i}} L_{\mathrm{s}}$ and the second is most important for smaller scales $l<\beta_{\mathrm{i}} L_{\mathrm{s}}$, i.e., the process in which the neutral atmosphere turbulence in conjunction with a mean (background) plasma density gradient produce plasma irregularities by mixing regions of high and low density dominates at the larger scales, while the interaction of the plasma imbedded in the turbulent motions of neutral gas with the magnetic field is more important for generation of the small-scale fluctuations.

\section{Spectrum of irregularities}

As mentioned in the previous section, the velocity of neutrals $\mathbf{u}(\mathbf{x}, t)$ and the plasma density $n(\mathbf{x}, t)$ can be 
expressed as sums of their regular and fluctuating components or, in other words, mean $\left(\mathbf{u}_{0}=\langle\mathbf{u}\rangle, n_{0}=\right.$ $\langle n\rangle)$ and random $\left(\left\langle\mathbf{u}_{1}\right\rangle=0,\left\langle n_{1}\right\rangle=0\right)$ ingredients. The velocity field $\mathbf{u}_{1}(\mathbf{x}, t)$ represents the neutral atmosphere turbulence which is similar to usual fluid turbulence.

Before considering the consequences of Eq. (11), we must digress briefly to recall certain basic properties of the random velocity field $\mathbf{u}_{1}(\mathbf{x}, t)$ that is statistically homogeneous in $\mathbf{x}$ and stationary in $t$ (see Batchelor, 1953; Tatarskij, 1967). The two-point correlation tensor of the field $\mathbf{u}_{1}(\mathbf{x}, t)$ is

$$
\begin{gathered}
\left\langle u_{1 i}(\mathbf{x}, t) \cdot u_{1 j}\left(\mathbf{x}^{\prime}, t^{\prime}\right)\right\rangle=R_{i j}(\mathbf{r}, \tau), \\
\mathbf{x}=\mathbf{x}^{\prime}-\mathbf{r}, \quad t=t^{\prime}-\tau .
\end{gathered}
$$

This quantity can be expressed in terms of the Fourier transform

$R_{i j}(\mathbf{r}, \tau)=\int \mathrm{d} \mathbf{k} \mathrm{d} \omega \Phi_{i j}(\mathbf{k}, \omega) \exp (\mathrm{ikr}-\mathrm{i} \omega \tau)$,

where $\Phi_{i j}$ is the spectrum tensor of the field $\mathbf{u}_{1}(\mathbf{x}, t)$. The relation inverse to Eq. (13) is

$\Phi_{i j}(\mathbf{k}, \omega)=(2 \pi)^{-4} \int \mathrm{d} \tau \mathrm{d} \mathbf{r} R_{i j}(\mathbf{r}, \tau) \exp (\mathrm{i} \omega \tau-\mathrm{ikr})$.

In practice, the assumption of exponential relaxation of the $\mathbf{u}$-field correlations in time is often valid and it gives

$R_{i j}(\mathbf{r}, \tau)=\int \mathrm{d} \mathbf{k} F_{i j}\left(\mathbf{k}\left(\exp \left(-|\tau| / \tau_{\mathbf{k}}\right) \exp (\mathrm{ikr})\right.\right.$,

where $\tau_{\mathrm{k}}$ is the "life" time of turbulent eddy with scale $|\mathbf{k}|^{-1}$. In the case of isotropic turbulence in the incompressible fluid the tensor $F_{i j}(\mathbf{k})$ is given by

$F_{i j}(\mathbf{k})=\left(\delta_{i j}-k_{i} k_{j} / k^{2}\right) E(k) / 4 \pi k^{2}, \quad k=|\mathbf{k}|$,

where $E(k)$ is the energy spectrum function. According to the universal equilibrium theory of Kolmogorov (1941), the energy spectrum function in the inertial range of wavenumbers is given on dimensional grounds by (see e.g., Batchelor, 1953)

$E(k)=C_{1} \varepsilon^{2 / 3} k^{-5 / 3} \quad\left(k_{0} \ll k \ll k_{\mu}\right)$,

where $C_{1}$ is a dimensionless constant of order unity $\left(C_{1} \approx 1.4\right), \varepsilon$ is the rate of flow of energy across any wave number magnitude $k$ in the inertial range, $k_{0}^{-1}=L_{0}$ is a length-scale of shear flow in which the kinetic energy of turbulence is generated, $k_{\mu}^{-1} \approx\left(\mu_{n}^{3} / \varepsilon\right)^{1 / 4}$ is a viscous length-scale at which say viscous dissipation is adequate to dissipate the energy at the rate $\varepsilon\left(\mu_{\mathrm{n}}\right.$ is the kinematic viscosity of neutral gas). The decay rate of eddy with scale $k^{-1}$ may be determined as a sum of the rate of eddy dissipation due to turbulent mixing, $\tau_{\mathrm{t}}^{-1}$, proper, and the rate of viscous dissipation, $\tau_{\mu}^{-1}$ :

$\tau_{\mathrm{k}}^{-1}=\tau_{\mathrm{t}}^{-1}+\tau_{\mu}^{-1}$,

$\tau_{\mathrm{t}}^{-1} \approx \varepsilon^{1 / 3} k^{2 / 3}$,

$\tau_{\mu}^{-1}=\mu_{\mathrm{n}} k^{2}$.

In the inertial range $\tau_{t}^{-1}>\tau_{\mu}^{-1}$, but the influence of viscosity cannot be neglected entirely for large wave numbers $k \rightarrow k_{\mu}$ (e.g., see Townsend, 1976).
Taking into account Eqs. (14), (15), (16) the spectrum tensor $\Phi_{i j}(\mathbf{k}, \omega)$ is

$\Phi_{i j}(\mathbf{k}, \omega)=\pi^{-1}\left(\delta_{i j}-k_{i} k_{j} / k^{2}\right) \frac{\tau_{\mathrm{k}}^{-1} E(k)}{4 \pi k^{2}\left(\omega^{2}+\tau_{\mathrm{k}}^{-2}\right)}$.

Under the assumption that random fields $\mathbf{u}_{1}(\mathbf{x}, t)$ and $\delta n(\mathbf{x}, t)$ are stationary function of $\mathbf{x}$ and $t$ with Fourier transforms

$u_{1 j}(\mathbf{x}, t)=\int \mathrm{d} \mathbf{k} \mathrm{d} \omega u_{1 j}(\mathbf{k}, \omega) \exp (\mathrm{i} \mathbf{k} \mathbf{x}-\mathrm{i} \omega t)$,
$\delta n(\mathbf{x}, t)=\int \mathrm{d} \mathbf{k} \mathrm{d} \omega \delta n(\mathbf{k}, \omega) \exp (\mathrm{i} \mathbf{k} \mathbf{x}-\mathrm{i} \omega t)$,

the Fourier transform of Eq. (11) is then

$$
\begin{aligned}
& \left(D_{\mathrm{a}} k^{2}+\tau_{\mathrm{r}}^{-1}-\mathrm{i} \omega\right) \delta n(\mathbf{k}, \omega) \\
& \quad+\mathrm{i} k_{j} \int \mathrm{d} \mathbf{k}^{\prime} \mathrm{d} \omega^{\prime} \delta n\left(\mathbf{k}^{\prime}, \omega^{\prime}\right) \cdot u_{1 j}\left(\mathbf{k}-\mathbf{k}^{\prime}, \omega-\omega^{\prime}\right) \\
& \quad=-L_{\mathrm{s}}^{-1} \mathbf{g} \cdot \mathbf{u}_{1}(\mathbf{k}, \omega)-\mathrm{i} \beta_{\mathrm{i}} \mathbf{k}\left(\mathbf{u}_{1}(\mathbf{k}, \omega) \times \mathbf{b}\right) .
\end{aligned}
$$

The convolution term on the left-hand side of Eq. (21) represents the contribution of mode interactions in the process of plasma fluctuation generation. If we take it into account phenomenologically through the coefficient of turbulent diffusion $D_{\mathrm{t}}$, then

$$
\begin{aligned}
& \left(\left(D_{\mathrm{a}}+D_{\mathrm{t}}\right) k^{2}+\tau_{\mathrm{r}}^{-1}-\mathrm{i} \omega\right) \delta n(\mathbf{k}, \omega) \\
& \quad=-L_{\mathrm{s}}^{-1} \mathbf{g} \cdot \mathbf{u}_{1}(\mathbf{k}, \omega)-\mathrm{i} \beta_{\mathrm{i}} \mathbf{k}\left(\mathbf{u}_{1}(\mathbf{k}, \omega) \times \mathbf{b}\right) .
\end{aligned}
$$

In the lower ionosphere the Schmidt number is near unity, $\mu_{\mathrm{n}} \approx D_{\mathrm{a}}$, as in the case of most fluids and gases, hence, we may regard that $D_{\mathrm{t}} k^{2} \approx \tau_{\mathrm{t}}^{-1}$ and Eq. (23) becomes

$\delta n(\mathbf{k}, \omega)=\frac{-L_{\mathrm{s}}^{-1} \mathbf{g} \cdot \mathbf{u}_{1}(\mathbf{k}, \omega)-\mathrm{i} \beta_{\mathrm{i}} \mathbf{k}\left(\mathbf{u}_{1}(\mathbf{k}, \omega) \times \mathbf{b}\right)}{D_{\mathrm{a}} k^{2}+\tau_{\mathrm{t}}^{-1}+\tau_{\mathrm{r}}^{-1}-\mathrm{i} \omega}$,

or, since $\mathbf{k}\left(\mathbf{u}_{1} \times \mathbf{b}\right)=\mathbf{u}_{1}(\mathbf{b} \times \mathbf{k})$,

$\delta n(\mathbf{k}, \omega)=\frac{-L_{\mathrm{s}}^{-1} \mathbf{g}-\mathrm{i} \beta_{\mathrm{i}}(\mathbf{b} \times \mathbf{k})}{D_{\mathrm{a}} k^{2}+\tau_{\mathrm{t}}^{-1}+\tau_{\mathrm{r}}^{-1}-\mathrm{i} \omega} \cdot \mathbf{u}_{1}(\mathbf{k}, \omega)$.

The correlation function of plasma density fluctuations, $Q(\mathbf{r}, t)$, with its spectral representation under the assumption of statistical homogeneity and stationarity can be written as follows

$$
\begin{aligned}
& Q(\mathbf{r}, t)=\left\langle\delta n(\mathbf{x}, t) \cdot \delta n\left(\mathbf{x}^{\prime}, t^{\prime}\right)\right\rangle \\
&=\int \mathrm{d} \mathbf{k} \mathrm{d} \omega \Psi(\mathbf{k}, \omega) \exp (\mathrm{ikr}-\mathrm{i} \omega \tau), \\
& \mathbf{x}=\mathbf{x}^{\prime}-\mathbf{r}, \quad t=t^{\prime}-\tau .
\end{aligned}
$$

The relation inverse to Eq. (25) is

$\Psi(\mathbf{k}, \omega)=(2 \pi)^{-4} \int \mathrm{d} \tau \mathrm{d} \mathbf{r} Q(\mathbf{r}, t) \exp (\mathrm{i} \omega \tau-\mathrm{ikr})$.

Using known relations for the statistically homogeneous and stationary random fields (see Batchelor, 1953; Tatarskij, 1967) 
$\left\langle u_{1 i}(\mathbf{k}, \omega) \cdot u_{1 j}^{*}\left(\mathbf{k}^{\prime}, \omega^{\prime}\right)\right\rangle=\Phi_{i j}(\mathbf{k}, \omega) \delta\left(\mathbf{k}-\mathbf{k}^{\prime}\right) \delta\left(\omega-\omega^{\prime}\right)$,

$\left\langle\delta n(\mathbf{k}, \omega) \cdot \delta n^{*}\left(\mathbf{k}^{\prime}, \omega^{\prime}\right)\right\rangle=\Psi(\mathbf{k}, \omega) \delta\left(\mathbf{k}-\mathbf{k}^{\prime}\right) \delta\left(\omega-\omega^{\prime}\right)$,

where the $*$ denotes a complex conjugate, and taking Eq. (24b) into account we then obtain an expression relating $\Psi$ to $\Phi_{i j}$, namely,

$$
\begin{aligned}
\Psi(\mathbf{k}, \omega)= & \frac{\left(L_{\mathrm{s}}^{-1} g_{i}+\mathrm{i} \cdot \beta_{\mathrm{i}}(\mathbf{b} \times \mathbf{k})_{i}\right)\left(L_{\mathrm{s}}^{-1} g_{j}-\mathrm{i} \cdot \beta_{\mathrm{i}}(\mathbf{b} \times \mathbf{k})_{j}\right)}{\left(\omega^{2}+\left(D_{\mathrm{a}} k^{2}+\tau_{\mathrm{t}}^{-1}+\tau_{\mathrm{r}}^{-1}\right)^{2}\right)} \\
& \times \Phi_{i j}(\mathbf{k}, \omega) .
\end{aligned}
$$

Here $g_{i(j)}$ represents the $i(j)$ th component of the unit vector $\mathbf{g}$, and $(\mathbf{b} \times \mathbf{k})_{i(j)}=\varepsilon_{i(j) m n} b_{m} k_{n}, \varepsilon_{i m n}$ is the alternating tensor ( 0 for any two indices being equal, +1 for any even number of permutations, and -1 for any odd number). Substituting the expression for the spectrum tensor $\Phi_{i j}(\mathbf{k}, \omega)$ given by Eq. (21) into Eq. (26) then leads to

$$
\begin{aligned}
\Psi(\mathbf{k}, \omega)= & \pi^{-1} \tau_{\mathrm{k}}^{-1} \\
& \times \frac{\left(L_{\mathrm{s}}^{-2} k^{-2}(\mathbf{g} \times \mathbf{k})^{2}+\beta_{\mathrm{i}}^{2}(\mathbf{b} \times \mathbf{k})^{2}\right) E(k)}{\left(\omega^{2}+\left(D_{\mathrm{a}} k^{2}+\tau_{\mathrm{t}}^{-1}+\tau_{\mathrm{r}}^{-1}\right)^{2}\right)\left(\omega^{2}+\tau_{\mathrm{k}}^{-2}\right) 4 \pi k^{2}} .
\end{aligned}
$$

The spatial spectrum of plasma density fluctuations $P(\mathbf{k})$ (or the wavenumber spectrum) is defined by the zerotime-delay correlation function

$$
\begin{aligned}
& \left\langle\delta n(\mathbf{x}, t) \cdot \delta n\left(\mathbf{x}^{\prime}, t\right)\right\rangle \\
& \quad=Q\left(\mathbf{x}-\mathbf{x}^{\prime}, 0\right)=\int \mathrm{d} \mathbf{k} P(\mathbf{k}) \exp \left(\mathbf{i k}\left(\mathbf{x}-\mathbf{x}^{\prime}\right),\right.
\end{aligned}
$$

and $P(\mathbf{k})$ is related with $\Psi(\mathbf{k}, \omega)$ as

$P(\mathbf{k})=\int_{-\infty}^{\infty} \mathrm{d} \omega \Psi(\mathbf{k}, \omega)$.

After integration, we have

$$
P(\mathbf{k})=\frac{\left(L_{\mathrm{s}}^{-2} k^{-2}(\mathbf{g} \times \mathbf{k})^{2}+\beta_{\mathrm{i}}^{2}(\mathbf{b} \times \mathbf{k})^{2}\right) E(k)}{\left(\tau_{\mathrm{k}}^{-1}+\tau_{\mathrm{t}}^{-1}+\tau_{\mathrm{r}}^{-1}+D_{\mathrm{a}} k^{2}\right)\left(\tau_{\mathrm{t}}^{-1}+\tau_{\mathrm{r}}^{-1}+D_{\mathrm{a}} k^{2}\right) 4 \pi k^{2}},
$$

which is often called the three-dimensional power spectral density (PSD) of the fluctuations. Using Eq. (29) we may obtain the root-mean-square level of plasma density fluctuations $\left\langle\left(n_{1} / n_{0}\right)^{2}\right\rangle^{1 / 2}$,

$\left\langle\left(n_{1} / n_{0}\right)^{2}\right\rangle^{1 / 2}=\left\langle(\delta n)^{2}\right\rangle^{1 / 2}=\left(\int \mathrm{d} \mathbf{k} P(\mathbf{k})\right)^{1 / 2}$,

the integration over the surface of the sphere of radius $k$ in $\mathbf{k}$-space then gives

$$
\left\langle\left(n_{1} / n_{0}\right)^{2}\right\rangle=\left\langle(\delta n)^{2}\right\rangle=\int_{k_{1}}^{k_{2}} P_{1}(k) \mathrm{d} k,
$$

where

$$
P_{1}(k)=\frac{2\left(L_{\mathrm{s}}^{-2}+\beta_{\mathrm{i}}^{2} k^{2}\right) E(k)}{3\left(\tau_{\mathrm{k}}^{-1}+\tau_{\mathrm{t}}^{-1}+\tau_{\mathrm{r}}^{-1}+D_{\mathrm{a}} k^{2}\right)\left(\tau_{\mathrm{t}}^{-1}+\tau_{\mathrm{r}}^{-1}+D_{\mathrm{a}} k^{2}\right)} .
$$

It may be seen from Eq. (30) that $P_{1}(k) \mathrm{d} k$ is the contribution to the power level of relative plasma density fluctuations from the wave number range $(k, k+\mathrm{d} k)\left(P_{1}(k)\right.$ is the one-dimensional spectrum of the fluctuations). To estimate the rms fluctuation level we take the following values of parameters which are quite realistic for the $E_{s}$-layer and the region of its formation: $L_{\mathrm{s}} \sim 2 \cdot 10^{3} \mathrm{~m}, n_{0} \sim 2 \cdot 10^{10} \mathrm{~m}^{-3}, \beta_{\mathrm{i}}=\Omega_{\mathrm{i}} / v_{\mathrm{i}}$ $\sim 0.1, D_{\mathrm{a}} \sim 10^{2} \mathrm{~m}^{2} \mathrm{~s}^{-1}, \varepsilon \sim 0.35 \mathrm{~m}^{2} \mathrm{~s}^{-3}, \alpha_{\mathrm{r}} \sim 3 \cdot 10^{-13}$ $\mathrm{m}^{3} \mathrm{~s}^{-1}$. It is interesting that the data from rocket experiments have shown the presence of sporadic- $E$ irregularities having the spatial periods of approximately $75-270 \mathrm{~m}$ with $\mathrm{rms}$ density fluctuation level greater than 10\% (Rao and Smith, 1968), and from Eq. (30) for the plasma density fluctuations with the same scales (75$270 \mathrm{~m}$ ) under the mentioned values of parameters we obtain an rms level of about $5.4 \%$, which is not at variance with the experimental result.

\section{Discussion and conclusions}

The one-dimensional power spectrum $P_{1}(k)$ (or the three-dimensional spectrum $P(\mathbf{k})$ ) describes plasma fluctuations created by the neutral atmosphere turbulence in the wave number range $k_{\mu}>k>L_{\mathrm{s}}^{-1}$. It was noted that the effect of the magnetic field on the formation of plasma density fluctuations dominates at the smaller scales or at the larger wave numbers $k>\left(\beta_{\mathrm{i}} L_{\mathrm{s}}\right)^{-1}$, whereas the relative role of the mean plasma density gradient is more important in the generation of the large-scale fluctuations, $k<\left(\beta_{\mathrm{i}} L_{\mathrm{s}}\right)^{-1}$.

Consider first the part of the spectrum $P_{1}(k)$ that describes the short-wave-length plasma fluctuations. The expression Eq. (31) may be simplified and using Eq. (17) it then gives

$$
P_{1}(k) \approx \frac{2 \cdot \beta_{\mathrm{i}}^{2} \varepsilon^{2 / 3} k^{1 / 3}}{3\left(\tau_{\mathrm{k}}^{-1}+\tau_{\mathrm{t}}^{-1}+\tau_{\mathrm{r}}^{-1}+D_{\mathrm{a}} k^{2}\right)\left(\tau_{\mathrm{t}}^{-1}+\tau_{\mathrm{r}}^{-1}+D_{\mathrm{a}} k^{2}\right)},
$$

$$
k_{\mathrm{d}}>k>\left(\beta_{\mathrm{i}} L_{\mathrm{s}}\right)^{-1}
$$

(in the presence of magnetic field, the plasma density fluctuations can be created by the turbulent motions of weakly ionized gas even though the motions of neutrals are incompressible and the background plasma density gradient is absent). By equating the time scale of diffusion of plasma density irregularities

$\tau_{\mathrm{d}}=\left(D_{\mathrm{a}} k^{2}\right)^{-1}$

with the time scale of turbulent motions $\tau_{t}$ we can obtain the wave number $k_{\mathrm{d}}$ at which diffusion becomes most important:

$k_{\mathrm{d}} \approx\left(\varepsilon / D_{\mathrm{a}}^{3}\right)^{1 / 4}$.

The diffusive length-scale $k_{\mathrm{d}}^{-1}$ is analogous to the viscous length-scale $k_{\mu}^{-1}$ for the neutral atmosphere turbulence and in our case (weakly ionized plasma of the ionospheric $E$-region) $k_{\mathrm{d}}^{-1}$ is close to $k_{\mu}^{-1}$. 
Under the mentioned values of parameters which define $P_{1}(k)$, in the considered wave number interval (for length-scales $40 \mathrm{~m}<l<200 \mathrm{~m}$ ) the inequalities $\tau_{\mathrm{t}}^{-1}>\tau_{\mathrm{d}}^{-1}, \tau_{\mathrm{t}}^{-1}>\tau_{\mathrm{r}}^{-1}$ are valid, and the local power spectral density $P_{1}(k)$ may be expected to be proportional to

$P_{1}(k) \propto k^{-1}\left(k_{\mathrm{d}}>k>\left(\beta_{\mathrm{i}} L_{\mathrm{s}}\right)^{-1}\right)$.

The long-wavelength irregularities $\left(\left(\beta_{\mathrm{i}} L_{\mathrm{S}}\right)^{-1}>k>L_{\mathrm{s}}^{-1}\right)$ may be described by the spectrum Eq. (31) in the following form

$P_{1}(k)=\frac{2 \cdot L_{\mathrm{s}}^{-2} \varepsilon^{2 / 3} k^{-5 / 3}}{3\left(\tau_{\mathrm{k}}^{-1}+\tau_{\mathrm{t}}^{-1}+\tau_{\mathrm{r}}^{-1}+D_{\mathrm{a}} k^{2}\right)\left(\tau_{\mathrm{t}}^{-1}+\tau_{\mathrm{r}}^{-1}+D_{\mathrm{a}} k^{2}\right)}$,

$\left(\beta_{\mathrm{i}} L_{\mathrm{s}}\right)^{-1}>k>L_{\mathrm{s}}^{-1}$

(intensity of the plasma fluctuations is proportional to the mean plasma density gradient $\left|\nabla n_{0}\right| / n_{0}=$ $\left.L_{\mathrm{g}}^{-1} \approx L_{\mathrm{s}}^{-1}\right)$. For this wave number interval there are three subranges of wave numbers: $\left(\beta_{\mathrm{i}} L_{\mathrm{s}}\right)^{-1}>k>k_{\mathrm{r}}$, $k_{\mathrm{r}}>k>k_{\mathrm{rr}}$, and $k_{\mathrm{rr}}>k>L_{\mathrm{s}}^{-1}$, where $k_{\mathrm{r}}$ and $k_{\mathrm{rr}}$ are determined (in order of magnitude) by equating the time scale $\tau_{\mathrm{t}}$ with $\tau_{\mathrm{r}}$ and $2 \tau_{\mathrm{r}}$, respectively:

$k_{\mathrm{r}} \approx \varepsilon^{-1 / 2}\left(2 \alpha_{\mathrm{r}} N_{\mathrm{R}}\right)^{3 / 2}$,

$k_{\mathrm{rr}} \approx \varepsilon^{-1 / 2}\left(\alpha_{\mathrm{r}} N_{\mathrm{R}}\right)^{3 / 2}$

In the subrange $\left(\beta_{\mathrm{i}} L_{\mathrm{s}}\right)^{-1}>k>k_{\mathrm{r}} \quad$ (length-scales $200 \mathrm{~m}<l<450 \mathrm{~m})$, the inequalities $\tau_{\mathrm{t}}^{-1}>\tau_{\mathrm{d}}^{-1}$, $\tau_{\mathrm{t}}^{-1}>\tau_{\mathrm{r}}^{-1}$ remain valid, however, because of the increasing influence of the mean density gradient the spectrum $P_{1}(k)$ becomes

$P_{1}(k) \propto k^{-3}\left(\left(\beta_{\mathrm{i}} L_{\mathrm{s}}\right)^{-1}>k>k_{\mathrm{r}}\right)$.

For the wave numbers $k_{\mathrm{r}}>k>k_{\mathrm{rr}}$ (length-scales $450 \mathrm{~m}<l<1273 \mathrm{~m}$ ), the inequalities $\tau_{\mathrm{t}}^{-1}<\tau_{\mathrm{r}}^{-1}<2 \tau_{\mathrm{t}}^{-1}$ are satisfied, i.e., recombination should be taken into account, and then

$P_{1}(k) \propto k^{-7 / 3}\left(k_{\mathrm{r}}>k>k_{\mathrm{rr}}\right)$.

In the subrange $k_{\mathrm{rr}}>k>L_{\mathrm{s}}^{-1}$ (length-scales $1273 \mathrm{~m}<$ $l<2000 \mathrm{~m})$, recombination damping dominates $\left(\tau_{\mathrm{r}}^{-1}>2 \tau_{\mathrm{t}}^{-1}\right)$ and the expected dependence $P_{1}(k)$ is

$P_{1}(k) \propto k^{-5 / 3}\left(k_{\mathrm{rr}}>k>L_{\mathrm{s}}^{-1}\right)$.

Thus, the formation of mid-latitude sporadic- $E$ irregularities by the neutral atmosphere turbulence at the length-scales $l_{\mathrm{d}}<l<L_{\mathrm{s}}\left(l_{\mathrm{d}} \approx\left(D_{\mathrm{a}}^{3} / \varepsilon\right)^{1 / 4}\right)$ is a combined action of such processes as: on the one hand the interaction of the turbulent velocity field with the mean plasma density gradient and the geomagnetic field, and, on the other hand, recombination, diffusion and turbulent eddy decay which operate as dissipation processes. A relative role of these processes is different at different scales of irregularities and it depends on the parameters of the $E_{s}$-layer. For example, the role of the density gradient and, say, the effect of recombination increase with increasing irregularity length-scales. The irregular- ity spectrum must be sensitive to the changing effects of the mentioned processes. If we use a power-law form for the irregularity spectrum, then some departures from the power-law form with a constant index (or some changes in the spectral slope) may be found. To estimate the characteristic length-scales at which such departures must occur, the following expressions can be used

$l_{\beta}=\beta_{\mathrm{i}} L_{\mathrm{g}} \approx \beta_{\mathrm{i}} L_{\mathrm{s}}$,

$l_{\mathrm{r}} \approx \varepsilon^{1 / 2} /\left(2 \alpha_{\mathrm{r}} N_{\mathrm{R}}\right)^{3 / 2}$,

$l_{\mathrm{rr}} \approx \varepsilon^{1 / 2} /\left(\alpha_{\mathrm{r}} N_{\mathrm{R}}\right)^{3 / 2}$

(for the length-scales $l>l_{\beta}$ the role of the mean plasma density gradient becomes more important than the influence of the magnetic field, for $l>l_{\mathrm{r}}$ the influence of recombination appears, and for $l>l_{\text {rr }}$ recombination becomes the main dissipation process).

In the present case (for the concrete realization of parameters chosen in the previous section) when $l_{\mathrm{d}}<l_{\beta}<l_{\mathrm{r}}<l_{\mathrm{rr}}<L_{\mathrm{s}}$, there are four subranges of length-scales. Figure la shows the self-normalized local $\operatorname{PSD}\left(P_{1}(k) / P_{1}\left(L_{\mathrm{s}}^{-1}\right)\right)$ of the plasma density fluctuations in this case (solid line 1). There are some differences between changes in the spectral slope in Fig.1a and the expected slopes from Eqs. (34), (38), (39) and (40), because dimensions of the subranges are not large enough. However, the mentioned expressions allow at least the trend of changes in the spectral slope to be predicted. In general the present spectrum may be described by a power law $k^{-1.9}$.

For comparison with the case discussed, the selfnormalized spectrum of the fluctuations under another value of the mean plasma density $n_{0} \sim 10^{11} \mathrm{~m}^{-3}$ (other parameters take the same values as in the previous case) is also illustrated in Fig. 1a (solid line 2). In this case, Eq. (30) gives the rms level of the plasma density fluctuations with length-scales $75-270 \mathrm{~m}$ about $3 \%$, and because of $l_{\mathrm{r}} \approx l_{\mathrm{d}} \approx 40 \mathrm{~m}$ there are three subranges of scales (or wave numbers): $l_{\mathrm{d}}<l<l_{\text {rr }}(40-114 \mathrm{~m}$ ), $l_{\text {rr }}<l<l_{\beta}(114-200 \mathrm{~m})$ and $l_{\beta}<l<L_{\mathrm{s}}(200-2000 \mathrm{~m})$. In these subranges the expected dependence $P_{1}(k)$ is

$$
\begin{aligned}
& P_{1}(k) \propto k^{-1 / 3}\left(k_{\mathrm{d}}>k>k_{\mathrm{rr}}\right), \\
& P_{1}(k) \propto k^{1 / 3}\left(k_{\mathrm{rr}}>k>l_{\beta}^{-1}\right), \\
& P_{1}(k) \propto k^{-5 / 3}\left(l_{\beta}^{-1}>k>L_{\mathrm{s}}^{-1}\right) .
\end{aligned}
$$

Recombination is an important dissipation process for the whole range of scales $l_{\mathrm{d}}<l<L_{\mathrm{s}}$ in the present case, although the influence of diffusion provides a steeply sloped "tail" of the spectrum. In the whole range of scales $P_{1}(k) \propto k^{-1.35}$.

So far the ion composition of sporadic- $E$ was regarded as that of the normal $E$-region (i.e., $\mathrm{NO}^{+}$ and $\mathrm{O}_{2}^{+}$). However, it is known that most of the ions at the sporadic- $E$ peak are often metallic such as $\mathrm{Fe}^{+}$and $\mathrm{Mg}^{+}$(Young et al., 1967; Behnke and Vickrey, 1975; Whitehead, 1989). These ions have very long lifetimes in the $E$-region since the recombination reaction for the 

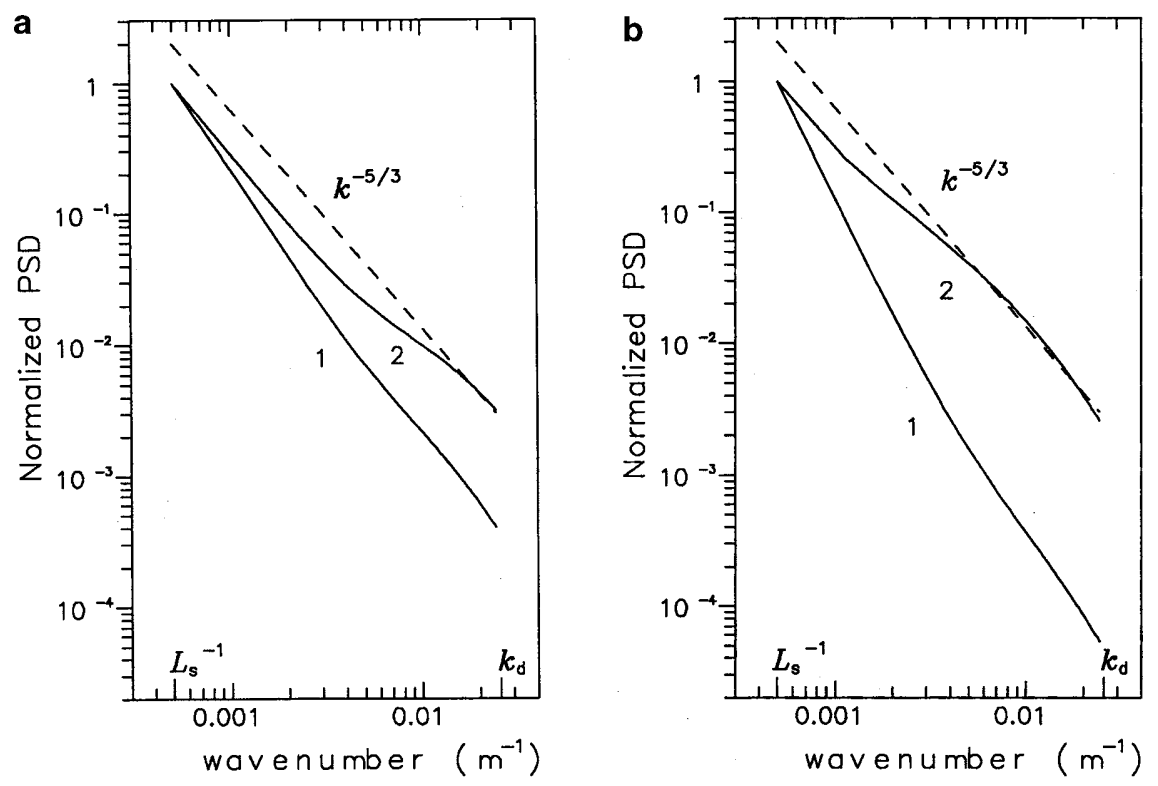

Fig. 1. a Self-normalized spectra of plasma density fluctuations predicted by Eq. (31) when $n_{0} \sim 2 \cdot 10^{10} \mathrm{~m}^{-3}$ (solid line 1 ) and $n_{0} \sim 10^{11} \mathrm{~m}^{-3}$ (solid line 2). The dashed line represents the slope of the Kolmogorov spectrum $k^{-5 / 3}$. b Self-normalized spectra of plasma density fluctuations predicted by Eq. (42) when $L_{\mathrm{g}} \approx L_{\mathrm{s}} \sim 2 \cdot 10^{3} \mathrm{~m}$ (solid line 1) and $L_{\mathrm{g}}>L_{\mathrm{s}}, L_{\mathrm{g}} \sim 2 \cdot 10^{4} \mathrm{~m}$ (solid line 2 )

monatomic ions is very slow. Hence, it is possible to suppose that $\tau_{\mathrm{r}}^{-1} \rightarrow 0$ in the expression for $P_{1}(k)$ and Eq. (31) then becomes

$P_{1}(k)=\frac{2\left(L_{\mathrm{g}}^{-2}+\beta_{\mathrm{i}}^{2} k^{2}\right) E(k)}{3\left(\tau_{\mathrm{k}}^{-1}+\tau_{\mathrm{t}}^{-1}+D_{\mathrm{a}} k^{2}\right)\left(\tau_{\mathrm{t}}^{-1}+D_{\mathrm{a}} k^{2}\right)}$.

There are two subranges of length-scales in this case: $l_{\mathrm{d}}<l<l_{\beta}(40-200 \mathrm{~m})$ where

$P_{1}(k) \propto k^{-1} \quad\left(k_{\mathrm{d}}>k>l_{\beta}^{-1}\right)$,

and $l_{\beta}<l<L_{\mathrm{g}} \approx L_{\mathrm{s}}(200-2000 \mathrm{~m})$ where

$P_{1}(k) \propto k^{-3} \quad\left(l_{\beta}^{-1}>k>L_{\mathrm{s}}^{-1}\right)$.

Figure $1 \mathrm{~b}$ (solid line 1) shows the self-normalized PSD predicted by Eq. (42) for the mentioned values of parameters, the rms level of density fluctuations having length-scales of approximately $75-270 \mathrm{~m}$ is about $7 \%$ in this case. In general the $k$-dependence of $P_{1}$ is close to $k^{-2.4}$. Since $L_{\mathrm{g}}^{-1}$ approaches zero (or at least $L_{\mathrm{g}} \gg L_{\mathrm{S}}$ ) near the $E_{s}$-layer peak where metallic ions dominate, the dependence predicted by Eq. (43) must be closer to the real situation. The PSD when, say, $L_{\mathrm{g}} \sim 2 \cdot 10^{4} \mathrm{~m}$ (i.e., $L_{\mathrm{g}}>L_{\mathrm{s}}$ ) is illustrated by solid line 2 in Fig. $1 \mathrm{~b}$, the rms fluctuation level is about $5.5 \%$. Because of the influence of diffusion for large wave numbers, the spectrum becomes close to $k^{-1.5}$ in this case.

Thus, the ion composition of sporadic- $E$ and the mean plasma density gradient exert apparent influence on the shape of the spectrum of plasma density fluctuations created by the neutral atmosphere turbulence. In addition, it is interesting that if the ion composition is unchanged throughout the $E_{s}$-layer then the rms fluctuation level when the fluctuation scales are not too large is higher on the sides of the layer where the mean density gradient is steeper than near the $E_{s}$ peak, on the other hand, if the slowly recombining ions dominate near the sporadic- $E$ peak while the usual $E$-region ion is the major one on the top and bottom of the layer then the fluctuation level is higher near the peak.

Of course, discussing the influence of the ion composition of sporadic- $E$ on the spectrum of irregularities, a mixture of ion species having different values of recombination coefficients should be considered. However, in spite of turbulent mixing the mentioned tendency of domination of the slowly recombining ions near the $E_{s}$ peak and the more rapidly recombining ones on the sides of the layer remains. Hence, in practice the spectrum of irregularities near the lower (or upper) boundary of the layer can be close to that given by Eq. (31) (in particular, its possible shape is shown as line 1 in Fig. 1a), and at the same time the irregularity spectrum near the $E_{s}$ peak can be close to that given by Eq. (42) (in Fig. 1b its shape may be shown as line 2). Probably, the spectrum in the intermediate region could be described by Eq. (31) with another value of the effective recombination coefficient $\left(0<\alpha_{\mathrm{r}}<3 \cdot 10^{-13}\right.$ $\mathrm{m}^{3} \mathrm{~s}^{-1}$ ), but this suggestion requires additional study.

Recently, the SEEK (Sporadic-E Experiment over Kyushu) campaign was conducted to investigate the mechanism for the generation of quasi-periodic radar backscatter from field-aligned irregularities imbedded in nighttime mid-latitude sporadic- $E$ layers. The SEEK was a coordinated field campaign, which included in-situ $E_{s}$ measurements using rockets and various groundbased measurements using radio and optical instruments, and has provided a large base of interesting data (Fukao et al., 1998). During the campaign a broad spectrum of plasma irregularities in the lower altitudes (90-115 km) was detected by sounding rocket-borne instruments (Pfaff et al., 1998; Mori and Oyama, 1998). The obtained spectra of irregularities had a power-law form, and a spectral index has changed from 0.8 to 1.2 for the plasma fluctuations in the lower part of the altitude range where an influence of neutral gas turbulence on the generation of ionospheric irregularities could take place (Mori and Oyama, 1998). It seems that 
spectral indices predicted have are consistent with those obtained during the SEEK campaign (unfortunately, a role of neutral turbulence in the formation of sporadic- $E$ irregularities was not considered in this campaign).

The attention of the present work has been focused on plasma irregularities caused by neutral turbulence in the mid-latitude sporadic- $E$. However, the approach developed here can be applied to the irregularities in the lower ionosphere at high and low latitudes. At least the present theoretical estimates of the spectral form and the rms level of plasma fluctuations do not seem to contradict experimental results obtained during rocket measurements in the auroral and equatorial lower ionosphere (see, e.g., Røyrvic and Smith, 1984; Pfaff et al., 1984, 1987a, b; Schlegel, 1992; Gurevich et al., 1996; Ulwick et al., 1993; Lübken et al., 1993). It should be noted that in some of the mentioned works (Pfaff et al., 1984, 1987a, b) plasma instabilities are proposed as basic mechanisms for the generation of ionospheric irregularities while in others a role of neutral turbulence in the formation of plasma irregularities is admitted (Røyrvic and Smith, 1984; Schlegel, 1992; Gurevich et al., 1996; Ulwick et al., 1993; Lübken et al., 1993).

The following conclusions can be made:

1 . In the mid-latitude sporadic- $E$, the neutral atmosphere turbulence can produce the plasma irregularities with length-scales from several tens of meters to several kilometers.

2. The spatial spectrum of such irregularities can be described by Eqs. (29), (31) or Eq. (42) when recombination can be ignored. According to these equations the irregularity spectrum in some cases is close to a powerlaw form, in other cases, however, some departures from simple power-law behavior can be found. These departures may be explained by the changing relative importance of the interaction of the turbulent velocity field with the geomagnetic field and with the mean vertical gradient in plasma density for the creation of irregularities of different length-scales, and, on the other hand, by changes in the relative role of such dissipation processes as diffusion, recombination and eddy decay in the turbulent cascade process. In other words, the shape of the irregularity spectrum depends on the sporadic- $E$ parameters.

3. The length-scales at which changes of the spectral slope must occur can be estimated from Eqs. (41).

4. A careful study of changes in the shape of the sporadic- $E$ irregularity spectrum and in the level of density fluctuations may provide information about the ion composition of the $E_{s}$-layer. For instance, a sufficiently high level of fluctuations near the $E_{s}$ peak (in comparison with the fluctuation level on the sides of the layer) can be used as a sign of domination of longlived ions there.

5. Expression (29) represents the three-dimensional PSD of the relative fluctuations in plasma density. According to Eq. (29), in general the irregularity spectrum is not quite isotropic due to the preferred directions of $\mathbf{b}$ and $\mathbf{g}$. These directions are given by the geomagnetic field, $\mathbf{b}$, and the mean plasma density gradient, $\mathbf{g}=L_{\mathrm{g}} n_{0}^{-1} \nabla n_{0}$, in the $E_{s}$-layer. Although it is not difficult to show that deviation of the spectrum (for plasma fluctuation scales within the inertial range of neutral turbulence) from isotropy is sufficiently weak, this deviation seems to be of interest and its detailed treatment is proceeding.

6 . In the case of the mid-latitude sporadic- $E$, the rms density fluctuation level predicted by Eq. (30) is in good agreement with the observed values (Rao and Smith, 1968). The shape of irregularity spectrum Eq. (31) (or Eq. 42) is in reasonable agreement with the spectra obtained from experimental data (Ovezgeldyev et al., 1988; Mori and Oyama, 1998) too. Nevertheless, it is difficult to be definite about a decisive corroboration of the present results because experimental data of midlatitude sporadic- $E$ irregularity observations are rather limited. To test our predictions here an experiment that measures simultaneously the irregularity spectrum and the pertinent parameters of the $E_{s}$-layer and the neutral atmosphere turbulence is needed. Since, for this purpose, rocket measurements are most suitable, experience obtained during the combined rocket and radar campaigns: the Condor (Pfaff et al., 1987a, b), the ROSE (Rose et al., 1992), the ERRIS (Pfaff et al., 1992), and the SEEK (Fukao et al., 1998), for example, is useful. In addition, it is important to make corrections of the operating frequencies for radar measurements (altering to the frequencies lower than VHF) taking into account results by Gurevich et al. (1997) and Schlegel and Gurevich (1997), and to use the transparency of sporadic- $E$ on ionograms as an indicator of turbulence and the height of the turbopause (Gorbunova and Shved, 1984; Whitehead, 1989).

7. As regards further investigations, an elimination of some simplifications can improve the present results. In particular, these include such things as ignoring the height dependence of several parameters (e.g., the temperature, the collision frequency, the recombination rate, etc.) within the layer and assuming the linear relation between the plasma density fluctuations and the turbulent velocity field of neutrals.

8. Although the role of plasma instabilities in the generation of the mid-latitude sporadic- $E$ irregularities was beyond our scope it should be said that the neutral atmosphere turbulence may probably play an important role in seeding the plasma processes, particularly in that case, when the turbulence in the $E$-region is stratified. A very thin layer of turbulence would lead to a $E_{s}$-layer with an almost square electron (plasma) density profile in the region of turbulence, falling off in a Gaussian fashion outside the eddy region (Chimonas, 1974), that could provide conditions for the gradient drift instability on the top and bottom of the mid-latitude sporadic- $E$ (Ecklund et al., 1981).

Thus, additional experimental and theoretical studies are needed to gain a better understanding of the processes of irregularity creation in the mid-latitude sporadic- $E$.

Acknowledgements. The author would like to thank S.F. Nosov for fruitful and stimulating discussions during the course of the work and J.D. Whitehead for helpful and important comments on the manuscript. The author also thanks the referees for the useful comments and suggestions that helped to improve the paper. 
Topical Editor M. Lester thanks K. Schlegel and another referee for their help in evaluating this paper.

\section{References}

Axford, W. I., The formation and vertical movement of dense ionized layers in the ionosphere due to neutral wind shears, J. Geophys. Res., 68, 769, 1963.

Batchelor, G. K., The theory of homogeneous turbulence, Cambridge University Press, London, 1953.

Behnke, R. A., and J. F. Vickrey, Radar evidence for $\mathrm{Fe}^{+}$in a sporadic E-layer, Radio Sci., 10, 325, 1975.

Chimonas, G., Turbulent diffusion as a controlling factor in sporadic-E, J. Atmos. Terr. Phys., 36, 235, 1974.

Cho, J. Y. N., W. E. Swartz, M. C. Kelley, and C. A. Miller, CUPRI observations of PMSE during Salvo B of NLC-91: evidence of both partial reflection and turbulent scatter, Geophys. Res. Lett., 20, 2291, 1993.

Ecklund, W. L., D. A. Carter, and B. B. Balsley, Gradient drift irregularities in mid-latitude sporadic-E, J. Geophys. Res., 86, 858,1981

Farley, D. T., Theory of equatorial electrojet plasma waves: new developments and current status, J. Atmos. Terr. Phys., 47, 729, 1985.

Feer, B. G., and M. C. Kelley, Ionospheric irregularities, Rev. Geophys. Space Phys., 18, 401, 1980.

Fooks, G. F., Irregularities in the $E_{s}$ layer of the ionosphere, Nature, 190, 707, 1961.

Fukao, S., M. Yamamoto, R. T. Tsunoda, H. Hayakawa, and T. Mukai, The SEEK (Sporadic- $E$ experiment over Kyushu) campaign, Geophys. Res. Lett., 25, 1761, 1998.

Gershman, B. N., Y. A. Ignatiev, and G. H. Kamenetskaya, Formation mechanisms of ionospheric sporadic layer $E_{s}$ on different latitudes (in Russian), Nauka, Moscow, 1976.

Gorbunova, T. A., and G. M. Shved, An analysis of the semitransparency of the $E_{s}$-layer as an indicator of turbulence under dynamically uniform conditions, Geomagn. Aeron., 24, 25, 1984.

Gurevich, A. V., N. D. Borisov, and K. P. Zybin, Ionospheric turbulence induced in the lower part of the $E$ region by the turbulence of the neutral atmosphere, J. Geophys. Res., 102, 379, 1997.

Gurevich, A. V., K. Rinnert, and K. Schlegel, Low frequency fluctuations in the lower E-region described by neutral turbulence, in Plasma instabilities in the ionospheric E-region, Ed. K. Schlegel, Cuvillier, Göttingen, 1996.

Haldoupis, C., A review of radio studies of auroral $E$ region ionospheric irregularities, Ann. Geophysicae, 7, 239, 1989.

Haldoupis, C., and K. Schlegel, A 50-MHz radio Doppler experiment for midlatitude $E$ region coherent backscatter studies: system description and first results, Radio Sci., 28, 959, 1993.

Haldoupis, C., D. T. Farley, and K. Schlegel, Type 1 echoes from the mid-latitude E-region ionosphere, Ann. Geophysicae, 15, 908, 1997.

Hamza, A. M., and J.-P. St-Maurice, A turbulent theoretical framework for the study of current-driven E region irregularities at high latitudes: basic derivation and application to gradient-free situations, J. Geophys. Res., 98, 11 587, 1993a.

Hamza, A. M., and J.-P. St-Maurice, A self-consistent fully turbulent theory of auroral $E$ region irregularities, J. Geophys. Res., 98, 11 601, 1993b.

Hamza, A. M., and R. N. Sudan, Subgrid modeling of convective turbulence in weakly ionized collisional plasma by renormalization group analysis, J. Geophys. Res., 100, 3669, 1995.

Huang, C.-M., E. Kudeki, S. J. Franke, C. H. Liu, and J. Röttger, Brightness distribution of midlatitude $E$ region echoes detected at the Chung-Li VHF radar, J. Geophys. Res., 100, 14 703, 1995.

Justus, C. G., The spectrum and scales of upper atmospheric turbulence, J. Geophys. Res., 72, 1933, 1967.
Kolmogorov, A. N., The local structure of turbulence in incompressible viscous fluid for very large Reynolds numbers, Dokl. AN SSSR, 30, 299, 1941.

Lübken, F.-J., G. Lehmacher, T. Blix, U.-P. Hoppe, E. Thrane, J. Cho, and W. Swartz, First in-situ observations of neutral and plasma density fluctuations within a PMSE layer, Geophys. Res. Lett., 20, 2311, 1993.

McComb, W. D., M. J. Filipiak, and V. Shanmugasundaram, Rederivation and further assessment of the LET theory of isotropic turbulence, as applied to passive scalar convection, J. Fluid Mech., 245, 279, 1992.

Mori, H., and K.-I. Oyama, Sounding rocket observation of sporadic-E layer electron-density irregularities, Geophys. Res. Lett., 25, 1785, 1998.

Namboothiri, S. P., A. H. Manson, and C. E. Meek, Extension of MF radar tidal measurements to $E$-region heights $(95-125 \mathrm{~km})$ : Saskatoon (52 N, 107 W), Canada, Ann. Geophysicae, 12, 333, 1994.

Nielsen, E., and K. Schlegel, Coherent radar Doppler measurements and their relationship to the ionospheric electron drift velocity, J. Geophys. Res., 90, 3498, 1985.

Ovezgeldyev, O. G., Y. Karadzhaev, and L. P. Korsunova, The amplitude spectrum of a radio signal reflected from the $E_{S}$-layer, Geomagn. Aeron., 28, 1024, 1988.

Pfaff, R. F., M. C. Kelly, B. G. Fejer, E. Kudeki, C. W. Carlson, A. Pedersen, and B. Hausler, Electric field and plasma density measurements in the auroral electrojet, J. Geophys. Res., 89, 236-244, 1984.

Pfaff, R. F., M. C. Kelly, E. Kudeki, B. G. Fejer, and K. D. Baker, Electric field and plasma density measurements in the strongly driven daytime equatorial electrojet. 1. The unstable layer and gradient drift waves, J. Geophys. Res., 92, 13 578, 1987a.

Pfaff, R. F., M. C. Kelly, E. Kudeki, B. G. Fejer, and K. D. Baker, Electric field and plasma density measurements in the strongly driven daytime equatorial electrojet. 2. Two-stream waves, J. Geophys. Res., 92, 13 597, 1987b.

Pfaff, R. F., J. Sahr, J. F. Providakes, W. E. Swartz, D. T. Farley, P. M. Kintner, I. Häggström, A. Hedberg, H. Opgenoorth, G. Holmgren, A. McNamara, D. Wallis, B. Whalen, A. Yau, S. Watanabe, F. Creutzberg, P. Williams, E. Nielsen, K. Shlegel, and T. R. Robinson, The $E$-region rocket/radar instability study (ERRIS): scientific objectives and campaign overview, J. Atmos. Terr. Phys., 54, 715, 1992.

Pfaff, R. F., M. Yamamoto, P. Marionni, H. Mori, and S. Fukao, The SEEK (Sporadic-E experiment over Kyushu) campaign, Geophys. Res. Lett., 25, 1769, 1998.

Ponyatov, A. A., The spectrum of the gradient-drift instability in the mid-latitude ionospheric E region, Geomagn. Aeron., 28, 33, 1988.

Rao, M. M., and L. G. Smith, Sporadic- $E$ classification from rocket measurements, J. Atmos. Terr. Phys., 30, 645, 1968.

Riggin, D., W. E. Swartz, J. F. Providakes, and D. T. Farley, Radar studies of long wavelength waves associated with mid-latitude sporadic E layers, J. Geophys. Res., 91, 8011, 1986.

Robinson, T. R., Towards a self-consistent nonlinear theory of radar auroral backscatter, J. Atmos. Terr. Phys., 48, 417, 1986.

Ronchi, C., R. N. Sudan, and D. T. Farley, Numerical simulations of large-scale plasma turbulence in the daytime equatorial electrojet, J. Geophys. Res., 96, 21 263, 1991.

Rose, G., K. Schlegel, K. Rinnert, H. Kohl, E. Nielsen, G. Dehmel, A. Friker, F.-J. Lübken, H. Lühr, E. Neske, and A. Steinweg, The ROSE project. Scientific objectives and discussion of first results, J. Atmos. Terr. Phys., 54, 657, 1992.

Rosenberg, N. W., and C. G. Justus, Space and time correlations of ionospheric winds, Radio Sci., 1, 149, 1966.

Ryrvic, O., and L. G. Smith, Comparison of mesospheric VHF radar echoes and rocket probe electron concentration measurements, J. Geophys. Res., 89, 9014, 1984.

Schlegel, K., Measurements of electron density fluctuations during the ROSE rocket flights, J. Atmos. Terr. Phys., 54, $715,1992$. 
Schlegel, K., and A. V. Gurevich, Radar backscatter from plasma irregularities of the lower $E$ region induced by neutral turbulence, Ann. Geophysicae, 15, 870, 1997.

Schlegel, K., and C. Haldoupis, Observation of the modified twostream plasma instability in the midlatitude $E$ region ionosphere, J. Geophys. Res., 99, 6219, 1994.

Schlegel, K., and H. Thiemann, Particle-in-cell plasma simulations of the modified two stream instability, Ann. Geophysicae, 12, $1091,1994$.

Smith, L. G., Rocket observations of sporadic $E$ and related features of the $E$ region, Radio Sci., 1, 178, 1966.

Sudan, R. N., Unified theory of type I and type II irregularities in the equatorial electrojet, J. Geophys. Res., 88, 4853, 1983.

Sudan, R. N., and D. Pfirsch, On the relation between "mixing length" and "direct interaction approximation" theories in twodimensional plasma turbulence, Phys. Fluids, 28, 1702, 1985.

Tatarskij, V. I., Wave propagation in the turbulent atmosphere (in Russian), Nauka, Moscow, 1967.

Teptin, G. M., and Y. M. Stenin, On the spectrum of electron concentration inhomogeneities in the lower ionosphere, Dokl. AN SSSR, 233, 85, 1977.
Townsend, A. A., Turbulent shear flow (2nd edn.), Cambridge University Press, Cambridge, 1976.

Ulwick, J. C., M. C. Kelly, C. Alcala, T. A. Blix, and E. V. Thrane, Evidence for two structuring and scattering mechanism and the associated role of aerosols in the polar summer mesosphere, Geophys. Res. Lett., 20, 2307, 1993.

Whitehead, J. D., The formation of the sporadic- $E$ layer in the temperate zones, J. Atmos. Terr. Phys., 20, 49, 1961.

Whitehead, J. D., Production and prediction of sporadic E, Rev. Geophys. Space Phys., 8, 65, 1970.

Whitehead, J. D., Recent work on mid-latitude and equatorial sporadic-E, J. Atmos. Terr. Phys., 51, 401, 1989.

Whitehead, J. D., Modeling of instabilities in mid-latitude sporadic- $E$, Proceedings of COSPAR Colloquium on Low-Latitude Ionospheric Physics, Taipei, Taiwan, 195, 1993.

Woodman, R. F., M. Yamamoto, and S. Fukao, Gravity wave modulation of gradient drift instabilities in mid-latitude sporadic E irregularities, Geophys. Res. Lett., 18, 1197, 1991.

Young, J. M., C. Y. Johnson, and J. C. Holmes, Positive ion composition of a temporal-latitude sporadic $E$ layer as observed during a rocket flight, J. Geophys. Res., 72, 1473, 1967. 